\title{
Research of Core Configuration File for Integrated SSH Framework Yongchang Ren ${ }^{1, a}$, Houhua Shen ${ }^{2, b}$ \\ ${ }^{1}$ College of Information Science and Technology, Bohai University, Jinzhou, 121013, China \\ ${ }^{2}$ College of Engineering, Bohai University, Jinzhou, 121013, China \\ arycryc@sina.com, bshenhouhuashh@126.com
}

Keywords: SSH framework; web.xml; struts.xml; applicationContext.xml; hibernate.cfg.xml

\begin{abstract}
SSH is a powerful open source framework of Java Web and a lightweight solution for develop J2EE enterprise-class Web application. For the characteristics of SSH framework configuration file content more, configuration complexity and difficult to grasp, study the core configuration files. Based on the study of web.xml file that integrated MVC framework and Web applications, research the configuration techniques and methods such as the core configuration file struts.xml of Struts framework, the core configuration file Context.xml of Spring Framework and the core configuration file hibernate.cfg.xml of Hibernate framework. This study enables developers to clearly understand the way to write the core configuration file of SSH framework, and contribute to team members work in parallel and improves development efficiency.
\end{abstract}

\section{Introduction}

Framework is the reusable design of entire system or part system, constituted by a set of abstract classes and the way of interaction between instances. The development of large enterprise-class Web application system usually requires good software architecture to facilitate the collaborative development expansion and upgrading. With Web technology become the de facto standard of display and manipulate data, enterprise use J2EE platform for integration of original distributed subsystem. As the good openness and scalability, J2EE has become the de facto standard for building Web application. At present the more popular open source framework SSH, is a lightweight solution to develop the Web applications of enterprise-class J2EE. For the developers using SSH framework integration, need to master a number of configuration files. And the process of program development is write configuration files constantly. Combined with the practical experience of software development and teaching process for years and through the technical framework to explain the core configuration files, designed to make the readers have a clear understanding about the wording of configuration file and call relations, to improve development efficiency.

\section{Web.xml Configuration File}

Any MVC framework needs to integrate with Web application, which requires the web.xml file. Web.xml is the important configuration file that load information about the servlet to web application, plays function of initialization servlet, filter, etc. Typically, all of the MVC framework needs to load a core controller by Web application, servlet or filter become a good choice as they automatically load with web services using. For Struts2 framework needs to load FilterDispatcher that will load the application Struts 2 frameworks as long as the Web application responsible for loading FilterDispatcher.

Instead of an ordinary Servlet, Struts 2 designed core controller to Filter. Therefore, to allow Web application to load FilterDispacher, only configure FilterDispatcher in the web.xml file. web.xml configuration process show in Fig.1.

web.xml configuration process is as follows:

Step 1: File declaration and header description.

Step 2: The initialization of Spring container. If the web application has only one Spring 
configuration file that named "applicationContext.xml" and placed in the WEB-INF path of Web application, then only need to add a section in web.xml file. If it has multiple configuration files need to load, and then apply < context-param> elements to determine the name of configuration files. When Web application starts, complete the initialization of the Spring container immediately.

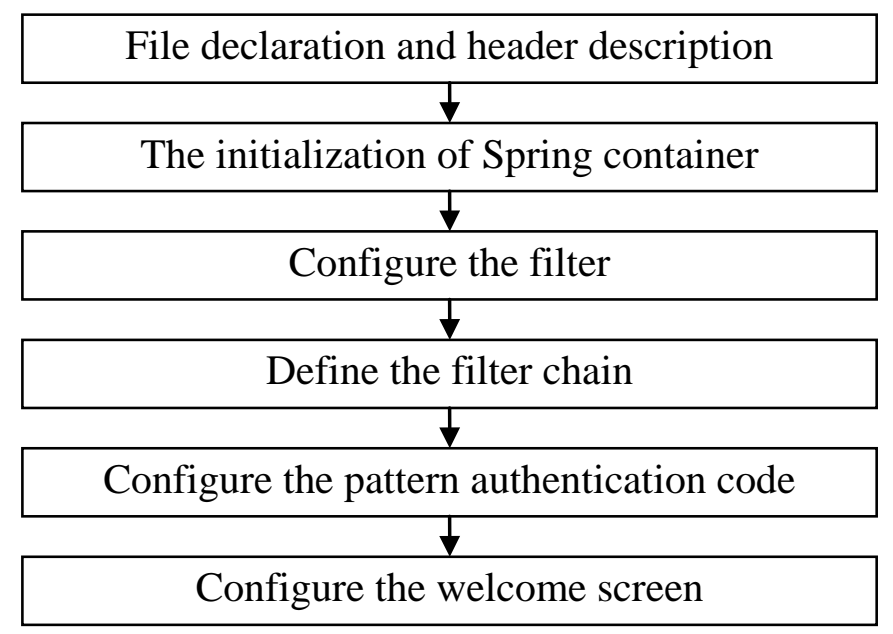

Fig.1 Web.xml configuration process

Step 3: Configure the filter. In web.xml file uses the following three filters, and the filter order cannot be changed. ActionContextCleanUp filter of Struts2, SiteMesh core filter and FilterDispathcer filter of Struts2 all are the core controllers of the MVC framework, responsible for intercepting all user's requests, and complete the initialization of the Struts2 framework.

Step 4: Define the filter chain. That means configure URL intercepted by Filter. Ranked No.1 is ActionContextCleanUp filter, ranked No.2 is SiteMesh core filter that map the core Filter of SiteMesh framework to filter all user's requests, ranked No.3 is FilterDispathcer filter.

Step 5: Configure the pattern authentication code. According to a global variable to determine whether there exist pattern authentication codes. If exist then configure, or not configure.

Step 6: Configure the welcome screen. Welcome page does not exist, then not configure. And configure any exist one.

\section{Struts Framework Configuration File}

Struts framework has two significant configuration files, struts.xml and struts.properties. Struts.xml responsible for the Action mapping of management application and its Result define. Struts properties file defines the global attributes of Struts framework. All the configuration files as shown in table 1 and the relationship between the configuration files as shown in Fig. 2.

Table 1 All configuration files of Struts framework

\begin{tabular}{c|l}
\hline File name & \multicolumn{1}{|c}{ Purpose } \\
\hline web.xml & Web deployment describer includes all of the necessary Struts components. \\
\cline { 1 - 2 } struts.xml & $\begin{array}{l}\text { The main configuration includes Result / View type, Action mapping, } \\
\text { block, etc. }\end{array}$ \\
\cline { 1 - 2 } struts.properties & The attribute configuration of Struts \\
\hdashline struts-defult.xml & The default configuration should be owned by xwork.xml \\
\hline velocity.properties & Velocity configuration \\
\hline struts-default.vm & The default configuration should be owned by velocity \\
\hline
\end{tabular}

The core configuration file of Struts framework is struts.xml that responsible for managing the business controller Action. By default, Struts framework automatically loads the struts.xml file which in WEB-INF/classes path. In most applications, as the increasing of apply scale the number of Action also increases that result to the configuration file struts.xml become very bloated. In order 
to avoid struts.xml file is too big, bloated and to improve the readability, can resolve it into multiple configuration files, and then include other configuration files into it.

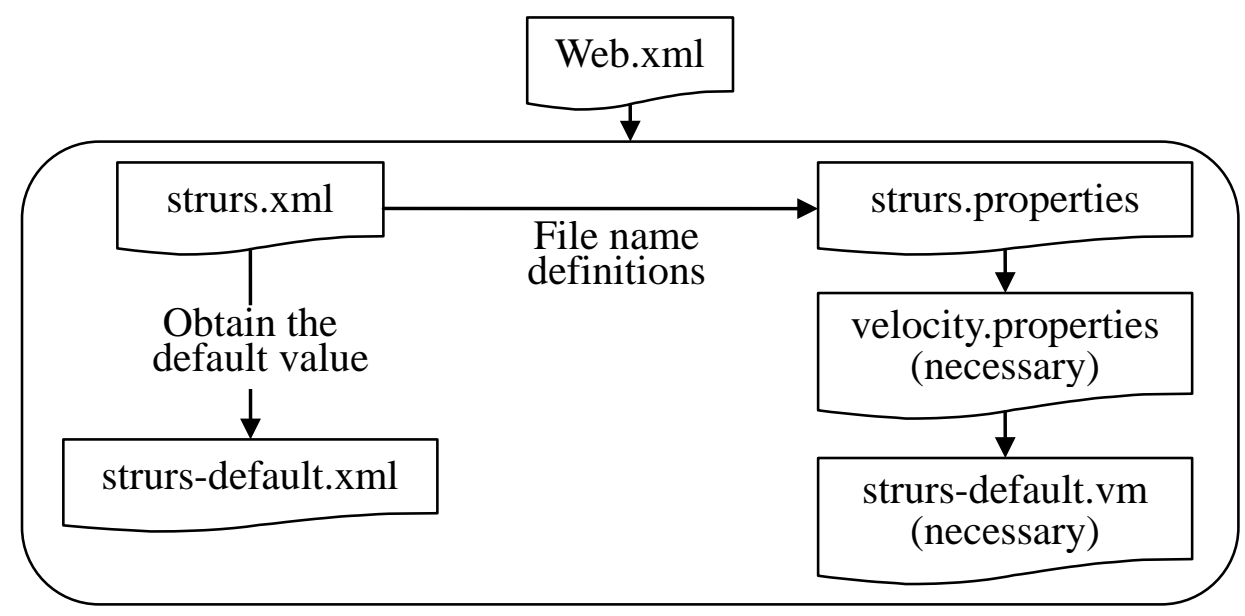

Fig. 2. The configuration file relational graph of Struts framework

\section{Spring Framework Configuration File}

Spring is a powerful java program framework, widely used in java programs, and use POJO to provide enterprise-class services. The core of Spring framework is the IoC (Inversion of Control) /DI (Dependency Injection) mechanism. Spring use the set value injection and constructor injection mechanism, through set up the collaboration object of configuration file management to construct IoC container that component can be created. The Spring framework construction shown in Fig.3.

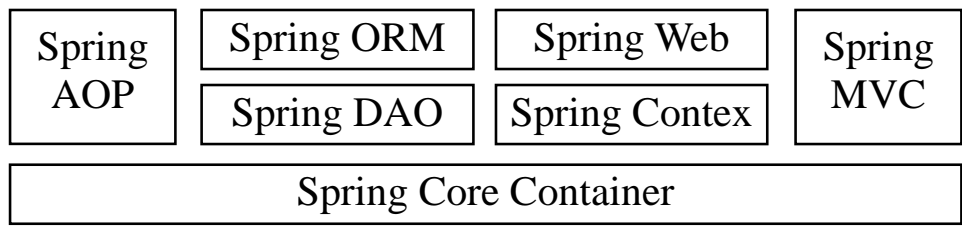

Fig.3. Spring framework construction

Spring uses dependency injection to obtain the simple and effective test capability. Spring beans, dependencies and the bean needed by service will be described in the configuration file which generally uses the XML format. Develop a large program, can set configuration file of spring into many. ApplicationContext.xml is the core configuration file, as it needs to set as much as content and XML configuration file lengthy and difficult to use, so large projects use a large number of bean will become difficult to read and control.

applicationContext.xml configuration techniques shown as follows:

(1) Make use of automatic assembly. According to the attribute name live matching type, bean attribute can be assembled automatically and constructed function can automatic assembly by match type.

(2) Use the simplified format. Simplified format helps to reduce redundancy, for it as the attribute values and quotes as attribute rather than child elements.

(3) Use type rather than index as much as possible to resolve the matching problem of the constructor parameters.

(4) Reuse bean definition. Use inheritance mechanism to reduce the repeat of configuration information and make the XML configuration simpler.

(5) Make use of the ApplicationContext rather than import to assemble bean.

(6) For each configuration file add a descriptive comment. In the XML configuration file best use id and name to describe rather than piles of notes.

(7) Communicate and change with team members. After modified java source codes, to make sure has changed the corresponding of configuration file and inform to team members. 
(8) Do not abuse injection. Not all of the java objects should be created through injection, overuse dependency injection will make the XML configuration more complex and lengthy.

\section{applicationContext.xml configuration steps shown as follows:}

Step1: File declaration and header description;

Step2: Define the data source Bean and using C3P0 data sources;

Step3: Define the SessionFactory of Hibernate;

Step4: Increase the configuration fragments of transaction control for business logic components in the Spring container. Including configure transaction manager Bean, configure transaction interceptor Bean and define BeanNameAutoProxyCreator post-processor;

Step5: Configure each Bean.

\section{Hibernate Framework Configuration File}

Hibernate is a mapping tool of object / relational database (Object / Relational Mapping) for Java environment. Object / Relational Mapping is a technique used to map the object of object model represented to a relational model database based SQL. In the software system the place of Hibernate between procedures and databases that formatted a date conversion platform of persistent objects and database tables. Hibernate architecture shown in Fig. 4.

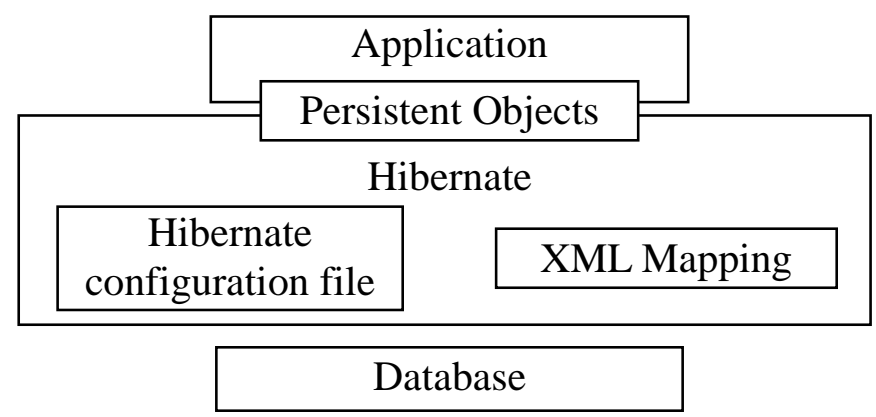

Fig.4. Hibernate architecture

As show as Fig. 4, Hibernate needs a configuration file to configure connection information of Hibernate and database and also needs an XML mapping file to determine the congruent relationship between persistent classes and tables and columns of data.

Hibernate configuration file is used to configure the parameters of Hibernate connection database and other variety of parameter values when it running. Hibernate configuration file not only can use the configuration way of traditional attribute file, but also can use configuration file of XML format. With the maturity and development of XML technology, using configuration file of XML format as the system's is more and more. The default name of XML format configuration file is hibernate.cfg.xml, and the attributes format configuration file's is hibernate.properties.

The main parameters of configuration file are as follows:

hibernate.connection.username: access user name of database;

hibernate.connection.password: access the password of database;

hibernate.connection.url: connect to the URL of database;

hibernate.connection.deiver_class: connect to the Driver used by database.

In the configuration file also includes other parameters:

hibernate.dialect: The Dialect used to connect to the database. Dialect is related with the type of used database, the role is to generate different SQL statements with different Dialect. There are some differences between SQL statements that supported by each databases, so a Dialect corresponds to a database and for the different databases to choose the right Dialect.

hibernate.show_sql: Display the SQL statements. To facilitate debugging, see the executed SQL statements in the console at any time. 
HBM file list: HBM file list can only be defined in the configuration file of XML format. When create Configuration object instance, it can make Hibernate automatically loading HBM files without manually loading by program.

\section{Conclusion}

Generally, framework has the reusability of plug and play, stability of maturity and good team cooperation. SSH framework is the typical three-tier structure based on J2EE technology. Business rules, data access, legitimacy verify and such work processed in the middle layer, customers does not directly interact with the database but establish a connection by the middle layer and then database interact from the middle layer. SSH framework enables developers to reduce the burden and energy of re-create solutions to complex problems program and extend to internal customized to adapt to technological upgrading and restructuring of reconstruction. All of these benefits reflected depend on a strong profile. This study content is the core technology of SSH framework and has a high reference value for developers in SSH framework.

\section{References}

[1] Li Gang, "Struts2 Definitive Guide," Publishing House of Electronic Industry, 2008.

[2] Li Gang, "Struts+Hibernate+Spring Detailed Application Development," Tsinghua University Press, 2007.

[3] Li Gang, "Struts+Hibernate+Spring Integrated Development Collection," Publishing House of Electronic Industry, 2007.

[4] Bai Gang-yuan, "Java Web development fully integrated self-study manual," China Machine Press, 2009.

[5] Li SH, Hu KH, Zhang FL, et al., "Implementation of Ssh2 Framework-based Management System for University Scientific Research Network," Computer Applications and Software, vol. 27, no. 7, pp. 195-196, 2010.

[6] Chen Y, Li S, Zhao YN, "Research and application of Web framework based on SSH+DWR," Journal of Nanjing University of Information Science \& Technology(Natural Science Edition), vol. 2, no. 5, pp. 455-460, 2010.

[7] Wu F, Ran WJ, "Framework based on J2EE architecture and its application SSH," China CIO News, vol. 23, no. 6, pp. 83-84, 2010.

[8] Wang Y, Tao ZK, "Design and Implementation of Code Generator Based on the SSH Framework," Transactions of Shenyang Ligong University, vol. 27, no. 1, pp. 25-27, 2008.

[9] Wang Y, Tao ZK, "The Applied Research of E-HR System Based on SSH," Journal of Wuhan University of Technology, vol. 31, no. 3, pp. 98-103, 2009. 